\title{
Evaluation of a chromogenic agar (MRSASelect) for the detection of meticillin-resistant Staphylococcus aureus with clinical samples in The Netherlands
}

Correspondence

I. H. M. van Loo

ihmvanloo@gmail.com

Received 20 October 2006

Accepted 1 December 2006

\author{
I. H. M. van Loo, S. van Dijk, I. Verbakel-Schelle and A. G. M. Buiting \\ Public Health Laboratory Tilburg, St Elisabeth Hospital, Tilburg, The Netherlands
}

\begin{abstract}
A novel chromogenic medium for the detection of meticillin-resistant Staphylococcus aureus (MRSA), MRSASelect (Bio-Rad), was evaluated with clinical samples in a public health laboratory in The Netherlands. In total, 3000 samples were tested in the period January to March 2005, including 972 nose, 972 throat, 968 perineum, and 88 wound or urine samples. Presumptive MRSA colonies appeared pink/mauve on the MRSASelect medium. The performance of MRSASelect medium was compared with the routine screening method. Evaluation of the colony morphology showed that all confirmed MRSA isolates grew as pink/mauve colonies. None of the white colonies were MRSA strains. The number of false-positive pink/mauve colonies increased after prolonged incubation from 20 to $48 \mathrm{~h}$. The specificity decreased from $92 \%$ after $20 \mathrm{~h}$ incubation to $89 \%$ after $48 \mathrm{~h}$ incubation. In total 70 MRSA strains were isolated, 55 of which were detected by the MRSASelect medium and 55 were detected by the routine screening method. Sensitivity was $78.6 \%$ for both test procedures, and specificities were 99.5 and $100 \%$, respectively for the MRSASelect medium and the routine screening method. The addition of an enrichment broth to the MRSASelect medium increased the number of MRSA strains detected by $12 \%$. In total, 18 patients were MRSA positive, 4 of these were detected by the MRSASelect medium only and 1 was detected by the routine screening method only. Sensitivity on patient level was 94.4 and $77.8 \%$ for the MRSASelect medium and the routine screening method, respectively, while specificities were 99.7 and $99.0 \%$.
\end{abstract}

\section{INTRODUCTION}

The prevalence of meticillin-resistant Staphylococcus aureus (MRSA) in The Netherlands is among the lowest in Europe; below $1 \%$ in both the community and in hospitals (Tiemersma et al., 2004; Wertheim et al., 2004). The Dutch search and destroy policy is part of the strategy to keep prevalence of MRSA low. Patients who are at risk for MRSA carriership are screened for colonization with it and isolated until screening cultures are negative, according to the guidelines established by the Dutch Working Group on Infection Prevention (http://www.wip.nl/UK/).

The routine screening procedure, used in this laboratory, takes at least $72 \mathrm{~h}$ to obtain a definitive result. Rapid detection of MRSA is essential to optimize therapy, to minimize patients' discomfort and to reduce costs. Chromogenic media are being developed to detect MRSA strains in one single step. Growth of non-staphylococcal strains is repressed by high salt concentration and enzymic substrate is used to achieve a specific colour reaction to

Abbreviation: MRSA, meticillin-resistant Staphylococcus aureus. detect $S$. aureus. Growth of meticillin-susceptible S. aureus strains is repressed by the addition of various combinations of antibiotics (Merlino et al., 2000). Various studies have been performed to evaluate different chromogenic media with clinical samples, two of which included the MRSASelect medium (Bio-Rad) (Becker et al., 2002; Ben Nsira et al., 2006; Blanc et al., 2003; Merlino et al., 2000; Perry et al., 2004; Simor et al., 2001; Stoakes et al., 2006). In this study, we evaluated the performance of a chromogenic MRSA agar, MRSASelect in a low prevalence population. We evaluated the MRSASelect medium by comparing it with our routine screening method on clinical samples. In the period January to March 2005 samples from known MRSA patients or patients at risk for MRSA carriership or contacts of known MRSA carriers were included in this study.

\section{METHODS}

Clinical samples. In total, 3000 samples from 409 patients were tested for the presence of MRSA in the Public Health Laboratory Tilburg, St Elisabeth Hospital, in the period January to March 2005. The laboratory provides diagnostics for two hospitals in Tilburg, and for physicians and nursing homes in the region. Samples were 
from patients who were at risk for MRSA carriership were follow up samples from known MRSA-positive patients or were samples from contacts of MRSA-positive patients to evaluate the spread of MRSA within the hospital. Cultures were taken from nose, throat, perineum, wounds, sputum and urine according to the guidelines of the Dutch Working Party on Infection Prevention. In total, 972 nose, 972 throat, 968 perineum and 88 wound or urine samples were included in the study.

Screening samples. Every sample was cultured on an MRSASelect plate (Bio-Rad) (composition of the medium is proprietary), a Columbia agar plate (Oxoid) supplemented with $5 \%$ sheep blood and $2 \mu \mathrm{g}$ ciprofloxacin $\mathrm{ml}^{-1}$ (BA-cipro) (Bayer), an MRSA screening agar, containing mannitol salt agar (Oxoid) supplemented with $4 \mu \mathrm{g}$ oxacillin $\mathrm{ml}^{-1}$ (MSA-oxa) (Sigma), and an enrichment broth containing Mueller-Hinton broth with $6 \mu \mathrm{g}$ oxacillin $\mathrm{ml}^{-1}$ and $6 \mu \mathrm{g}$ aztreonam $\mathrm{ml}^{-1}$ (MH-oxa-aztr) (ICN). For each sample the inoculated plates were analysed after $20 \mathrm{~h}$ and after $48 \mathrm{~h}$. The enrichment broth was subcultured after $20 \mathrm{~h}$ on a Columbia agar plate supplemented with $5 \%$ sheep blood, and analysed after $20 \mathrm{~h}$ and after $48 \mathrm{~h}$. The nose, throat and perineum samples from known MRSA patients were put into an enrichment broth separately, while unknown patients' nose, throat and perineum samples were pooled in one enrichment broth. If the pooled enrichment broth was positive the three samples were counted once. For the calculation of the sensitivity and specificity the samples from the solid media and the non-pooled enrichment broth were used.

Testing of colonies on MRSASelect medium. Presumptive MRSA colonies on the MRSASelect agar were pink/mauve and were tested with a latex agglutination test (Staphaurex plus, Murex Diagnostics) and subcultured on a Columbia agar plate supplemented with $5 \%$ sheep blood. If colony morphology was suspect for $S$. aureus and the latex agglutination test was positive further identification was performed (see below).

A total of 205 white colonies were tested with a latex agglutination test and subcultured on a Columbia agar plate supplemented with $5 \%$ sheep blood. Latex agglutination and catalase tests (Isenberg, 2004) were performed to exclude growth of $S$. aureus.

Confirmation of MRSA. Presumptive MRSA colonies (any colony on the Columbia agar plate and the MRSA screening agar, and pink/ mauve colonies on the MRSASelect plate) were identified by latex agglutination test, coagulase tube test (Isenberg, 2004), DNase (Isenberg, 2004), growth on a Mueller-Hinton plate supplemented with $6 \mu \mathrm{g}$ oxacillin $\mathrm{ml}^{-1}$, growth on a Mueller-Hinton plate supplemented with $2 \% \mathrm{NaCl}$, AccuProbe (Gen-Probe) and identification with the Vitek2 system (bioMérieux).

Susceptibility testing was performed by Vitek2 system. Isolates resistant ?for oxacillin, quinolones or aminoglycosides were further evaluated, including disc diffusion of oxacillin $(1 \mu \mathrm{g})$ and meticillin $(5 \mu \mathrm{g})$ (Sigma) on a Mueller-Hinton plate supplemented with $2 \%$ $\mathrm{NaCl}$, disc diffusion of ciprofloxacin $(5 \mu \mathrm{g})$, ofloxacin $(5 \mu \mathrm{g})$, mupirocin $(5 \mu \mathrm{g})$, gentamicin $(10 \mu \mathrm{g})$ and tobramycin $(10 \mu \mathrm{g})$ (Sigma) on a Mueller-Hinton plate and an E-test for oxacillin (AB Biodisk) on a Mueller-Hinton plate supplemented with $2 \% \mathrm{NaCl}$. Final confirmation was performed by mecA gene PCR (Del Vecchio et al., 1995).

\section{RESULTS AND DISCUSSION}

In this study a chromogenic medium, MRSASelect, was evaluated with clinical samples. First we evaluated the colonies that grew on the MRSASelect medium. After $20 \mathrm{~h}$ incubation 33 (89\%) MRSA strains were isolated and 4 (11\%) samples generated false-positive pink/mauve colonies (Table 1). Other studies evaluating MRSASelect medium found a lower number of false-positive samples, $3(3 \%)$ and $1(1 \%)$, respectively (Stoakes et al., 2006; Ben Nsira et al., 2006). We found that prolonging incubation from 20 to $48 \mathrm{~h}$ increased the yield of confirmed MRSA strains by $38 \%$ (20 strains) (Table 1). Ben Nsira et al. (2006) found an increase of $3 \%$. Stoakes et al. (2006) did not evaluate MRSASelect medium after 48 h. However, $97 \%$ of the MRSA strains were recovered after $18 \mathrm{~h}$ incubation, compared to $79 \%$ after $48 \mathrm{~h}$ in our study. For other chromogenic media the yield also increased after prolonged incubation. For example, Perry et al. (2004) found an increased yield of $11 \%, 19 \%$ and $20 \%$ for MRSA-ID, CHROMagar MRSA and ORSAB, respectively. Simor et al. (2001) also found an increase in recovery (23\%) of MRSA on ORSAB plates after prolonged incubation.

Table 1. Number and identification of pink/mauve and white colonies growing on MRSASelect after 20 and $48 \mathrm{~h}$ incubation

\begin{tabular}{|c|c|c|c|}
\hline & $\begin{array}{c}\text { No. of colonies } \\
\text { growing after } 20 \mathrm{~h}^{\star}\end{array}$ & $\begin{array}{c}\text { No. of colonies } \\
\text { growing after } 48 \mathrm{~h} \dagger\end{array}$ & Total \\
\hline \multicolumn{4}{|l|}{ Pink/mauve colonies } \\
\hline MRSA & 33 & 20 & 53 \\
\hline CNS & 1 & 2 & 3 \\
\hline Corynebacterium spp. & 3 & 4 & 7 \\
\hline Enterococci & 0 & 6 & 6 \\
\hline \multicolumn{4}{|l|}{ White colonies } \\
\hline CNS & 48 & 58 & 106 \\
\hline S. aureus & 0 & 1 & 1 \\
\hline
\end{tabular}

CNS, coagulase-negative staphylococci.

${ }^{\star}$ Number of pink/mauve or white colonies growing after $20 \mathrm{~h}$ on MRSASelect.

$\dagger$ Number of pink/mauve or white colonies growing after $48 \mathrm{~h}$ on MRSASelect that did not grow after $20 \mathrm{~h}$ incubation. 
Table 2. Number of MRSA-positive samples for the different media

\begin{tabular}{|lccc|}
\hline & $\begin{array}{c}\text { No. of MRSA- } \\
\text { positive samples }\end{array}$ & Sensitivity & Specificity \\
\hline MSA-oxa/BA-cipro & 37 & $52.9 \%$ & $100 \%$ \\
MSA-oxa/BA-cipro + MH-oxa-aztr & 55 & $78.6 \%$ & $100 \%$ \\
MRSASelect & 55 & $78.6 \%$ & $99.5 \%$ \\
MSA-oxa/BA-cipro + MH-oxa-aztr + MRSASelect & 70 & $100.0 \%$ & $100 \%$ \\
\hline
\end{tabular}

In total, 69 samples generated pink/mauve colonies after $48 \mathrm{~h}$ incubation, $53(77 \%)$ of which were true positives and $16(23 \%)$ were false positives (Table 1). Specificity decreased from $92 \%$ after $20 \mathrm{~h}$ incubation to $89 \%$ after 48 h. False positives were enterococci or coagulase-negative staphylococci (Table 1). Ben Nsira et al. (2006) found four $(4 \%)$ false positives after $48 \mathrm{~h}$. Compared to other chromogenic media (e.g. ORSAB medium) MRSASelect generated less false-positive results (Becker et al., 2002; Blanc et al., 2003; Simor et al., 2001). Other chromogenic media also show a decrease in specificity after $48 \mathrm{~h}$ incubation (Perry et al., 2004).

The colony morphology of false positives in our study showed less pigmentation compared to confirmed MRSA strains, an observation that was also noted by Ben Nsira et al. (2006). Similar observations were found in studies evaluating other chromogenic media (Becker et al., 2002; Blanc et al., 2003; Perry et al., 2004; Simor et al., 2001).

In total, 107 white colonies were evaluated, 106 of which were determined as coagulase-negative staphylococci and one of which as meticillin-susceptible S. aureus (Table 1). The number of white colonies doubled after prolonging incubation from 20 to $48 \mathrm{~h}$ (Table 1).

We compared MRSASelect medium with the routine screening method (MSA-oxa/BA-cipro + MH-oxa-aztr) (Table 2). Seventy confirmed MRSA isolates were found in eighteen patients (Table 2). Fifty-five MRSA isolates were detected by the screening method and fifty-five by the MRSASelect medium after $48 \mathrm{~h}$ incubation. Fifteen MRSA isolates were not detected by the MRSASelect medium and fifteen MRSA isolates were not detected by the routine screening method. Sensitivity of the MRSASelect medium was $78.6 \%$ and specificity was $99.5 \%$, compared to sensitivity of $78.6 \%$ and specificity of $100 \%$ for the routine screening method. The number of discrepancies between the MRSASelect medium and the routine screening method is unexpectedly high. We cannot explain the lower sensitivity in our study in comparison to the studies of Stoakes et al. (2006) and Nsara et al. (2006). Stoakes et al. (2006) found that 3 from the 111 confirmed MRSA strains were not detected by MRSASelect (sensitivity of $97.3 \%$ ) and Ben Nsira et al. (2006) reported 1 missing MRSA isolate (sensitivity $99.8 \%$ ). Differences in sampling, in MRSA prevalence and in circulating clones in the different countries may account for differences in sensitivity. The sensitivity we found was similar to that of other chromogenic media evaluated with clinical samples (Perry et al., 2004). To increase the yield we added an enrichment broth (MH-oxa-aztr) in a pilot study. From 225 samples we subcultured the enrichment broth on MRSASelect medium. We found $12 \%$ more MRSA isolates. We have now used the MRSASelect medium in combination with the enrichment broth (MH-oxa-aztr) in our laboratory for more then a year. Thirty percent of all the MRSA isolates were yielded from the enrichment broth, indicating an increase in yield that is higher than in the pilot study.

Analysis at the patient level showed that from a total of 408 patients screened for MRSA carriership 18 were MRSA carriers. MRSA isolates of 13 patients were detected by both methods. MRSA isolates of 4 carriers were detected by MRSASelect medium only, while 1 patient was detected by the routine screening method only. Sensitivity at the patient level was 94.4 and $77.8 \%$ for the MRSASelect medium and the routine screening method, respectively, while specificities were 99.7 and $99.0 \%$. The number of patients with MRSA that were missed in our study was $1 / 18$ (5\%) for MRSASelect and 4/18 (22\%) for the routine screening method. Perry et al. (2004) found similar figures for other chromogenic media: 2/43 (5\%), 7/43 (16\%) and 4/43 (9\%) for MRSA-ID, CHROMagar and ORSAB, respectively, and $12 / 43(28 \%)$ for mannitol salt agar.

In conclusion, in our hands MRSASelect is a useful medium for the detection of MRSA, although in this study it was less sensitive than in other studies. The MRSASelect medium generated a lower number of false positives compared to other chromogenic media. The increase in true-positive MRSA isolates after a prolonged incubation in our study is higher than for other chromogenic media. Optimization of screening was achieved by the addition of an enrichment broth next to primary culture on the MRSASelect medium. In a country with low MRSA prevalence (e.g. The Netherlands) the main advantage of MRSASelect medium compared to the routine screening procedure is that the time to obtain a negative culture result is reduced from 72 to $48 \mathrm{~h}$.

\section{REFERENCES}

Becker, A., Forster, D. H. \& Kniehl, E. (2002). Oxacillin resistance screening agar base for detection of methicillin-resistant Staphylococcus aureus. J Clin Microbiol 40, 4400-4401. 
Ben Nsira, S., Dupuis, M. \& Leclercq, R. (2006). Evaluation of MRSASelect, a new chromogenic medium for the detection of nasal carriage of methicillin-resistant Staphylococcus aureus. Int $J$ Antimicrob Agents 27, 561-564.

Blanc, D. S., Wenger, A. \& Bille, J. (2003). Evaluation of a novel medium for screening specimens from hospitalized patients to detect methicillin-resistant Staphylococcus aureus. J Clin Microbiol 41, 3499-3502.

Del Vecchio, V. G., Petroziello, J. M., Gress, M. J., McCleskey, F. K., Melcher, G. P., Crouch, H. K. \& Lupski, J. R. (1995). Molecular genotyping of methicillin-resistant Staphylococcus aureus via fluorophore-enhanced repetitive-sequence PCR. J Clin Microbiol 33, 2141-2144.

Isenberg, H. D. (editor) (2004). Clinical Microbial Procedures Handbook, 2nd edn. Washington, DC: ASM.

Merlino, J., Leroi, M., Bradbury, R., Veal, D. \& Harbour, C. (2000). New chromogenic identification and detection of Staphylococcus aureus and methicillin-resistant S. aureus. J Clin Microbiol 38, 2378-2380.

Perry, J. D., Davies, A., Butterworth, L. A., Hopley, A. L., Nicholson, A. \& Gould, F. K. (2004). Development and evaluation of a chromogenic agar medium for methicillin-resistant Staphylococcus aureus. J Clin Microbiol 42, 4519-4523.

Simor, A. E., Goodfellow, J., Louie, L. \& Louie, M. (2001). Evaluation of new medium oxacillin resistance screening agar base for the detection of methicillin-resistant Staphylococcus aureus from clinical specimens. J Clin Microbiol 39, 3422-3423.

Stoakes, L., Reyes, R., Daniel, J., Lennox, G., John, M. A., Lannigan, R. \& Hussain, Z. (2006). Prospective comparison of a new chromogenic medium, MRSASelect, to CHROMagar MRSA and mannitol-salt medium supplemented with oxacillin or cephoxitin for detection of methicillin-resistant Staphylococcus aureus. J Clin Microbiol 44, 637-639.

Tiemersma, E. W., Bronzwaer, S. L., Lyytikainen, O., Degener, J. E., Schrijnemakers, P., Bruinsma, N., Monen, J., Witte, W. \& Grundman, H. (2004). Methicillin-resistant Staphylococcus aureus in Europe, 1999-2002. Emerg Infect Dis 10, 1627-1634.

Wertheim, H. F., Vos, M. C., Boelens, H. A., Voss, A., Vandenbroucke-Grauls, C. M., Meester, M. H., Kluytmans, J. A., van Keulen, P. H. \& Verbrugh, H. A. (2004). Low prevalence of methicillin-resistant Staphylococcus aureus (MRSA) at hospital admission in the Netherlands: the value of search and destroy and restrictive antibiotic use. J Hosp Infect 56, 321-325. 